\title{
Conformation-Crooking CXCL4 to Unravel Autoimmune Heparin-Induced Thrombocytopenia
}

\author{
Rory R. Koenen ${ }^{1,2}$ \\ ${ }^{1}$ Department of Biochemistry, Cardiovascular Research Institute \\ Maastricht, Maastricht University, Maastricht, The Netherlands \\ 2 Institute for Cardiovascular Prevention, LMU Munich, Munich, \\ Germany
}

Thromb Haemost 2021;121:258-260.

Heparin-induced thrombocytopenia (HIT) is a clinically relevant adverse effect of heparin therapy, commonly used for thromboprophylaxis after, for example, (major) surgery ${ }^{1,2}$ or membrane oxygenation. ${ }^{3}$ Depending on the preparation of heparin, the incidence of HIT may range from 2 to 30 cases per 1,000 treatments. ${ }^{4}$ The main hallmark of HIT is a sudden severe drop in circulating platelet counts, typically 5 to 10 days after the initiation of (first-time) heparin treatment. Despite low platelet counts, HIT is paradoxically accompanied by venous, microvascular, and arterial thrombosis in up to $50 \%$ of cases $^{5}$ and often calls for alternative strategies of anticoagulation. ${ }^{2,6}$

The pathologic mechanism of HIT, known to date, is an immune reaction toward complexes of platelet factor 4 (CXCL4), a chemokine abundantly released from platelets, ${ }^{7}$ and negatively charged polymers (polyanions, e.g. heparin, nucleic acids, polyphosphates, bacterial cell wall components). Binding of CXCL4 to such polyanions may lead to the formation of neoepitopes in CXCL4. ${ }^{8,9}$ In addition, the concatenation of CXCL4 on the elongated anionic templates can induce the formation of large immunogenic aggregates with a high potential to initiate immune responses in $\mathrm{B}$ cells. ${ }^{10}$ The resulting antibodies directed against the CXCL4/polyanion complexes potently activate platelets and monocytes by crosslinking their gamma globulin (Fcr) receptors, ultimately leading to abnormal platelet and leukocyte counts, vascular inflammation, and thrombosis. ${ }^{2,11}$

In rare cases, HIT can also occur without any (prior) exposure to heparin. This spontaneous or autoimmune HIT might be triggered by major orthopaedic surgery or bacterial infections. The resulting clinical symptoms, low platelet counts and severe complications (disseminated intravascular coagulation, microvascular thrombosis), can persist for weeks. The antibodies that characterize autoimmune HIT can also activate platelets in the absence of heparin and appear to bind more strongly to CXCL4.

\section{received}

November 4, 2020

accepted

November 5, 2020

published online

December 31, 2020

Address for correspondence Rory R. Koenen, PhD, CARIM, Maastricht University, P.O. Box 616, 6200MD Maastricht, The Netherlands (e-mail: r.koenen@maastrichtuniversity.nl).

Deciphering the properties of antibodies that characterize and cause HIT may give cues to understanding HIT pathophysiology. For example, X-ray crystallography studies on the model monoclonal HIT-antibody "KKO" bound to the complex of CXCL4 with fondaparinux yielded knowledge about the nature of HIT antibody interactions and of their epitopes. ${ }^{9}$ However, the structural determinants of antibodies involved in (the heparin-independent) autoimmune HIT are still unknown. In addition, the characterization of antibodies from patient sera is hampered by difficult purification procedures, poor yield, and the polyclonal nature of the resulting antibody isolates.

In the study by Vayne et al in this issue of Thrombosis and Haemostasis, ${ }^{12}$ humanized monoclonal antibodies (humAbs) were raised against a complex of CXCL4 with heparin to serve as a model for the antibodies found in patients with autoimmune HIT. Since these antibodies contained a human Fc region, they behaved as HIT or autoimmune HIT antibodies, similar to those found in patients. Through biochemical and biophysical techniques, the authors characterized the properties of the three obtained humAbs and compared them with the existing model antibody KKO. All antibodies bound to immobilized CXCL4/heparin complexes to a similar extent and the concentration-dependent signal intensities of the humAbs resembled those of sera from patients with autoimmune HIT. Like KKO, the novel humAbs induced platelet activation. But unlike KKO, this also occurred in the absence of heparin, which is a feature typical of autoimmune HIT. Atomic force microscopy revealed high binding strengths and low off-rates of the humAbs, comparable to autoimmune HIT antibodies from patient sera. Isothermal titration calorimetry showed a notable release of heat when CXCL4 was titrated with the humAbs, which reflected the conformational change in CXCL4 exposing the neoepitope(s). This was supported by dynamic light scattering, where the humAbs effectively induced oligomerization of CXCL4, similar to the

(c) 2020. Thieme. All rights reserved. Georg Thieme Verlag KG,

Rüdigerstraße 14 ,

70469 Stuttgart, Germany
DOI https://doi.org/ 10.1055/s-0040-1721774. ISSN 0340-6245. 
A

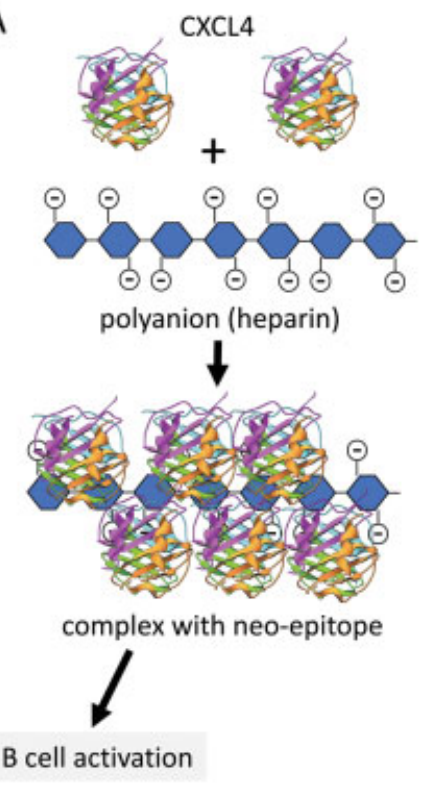

B

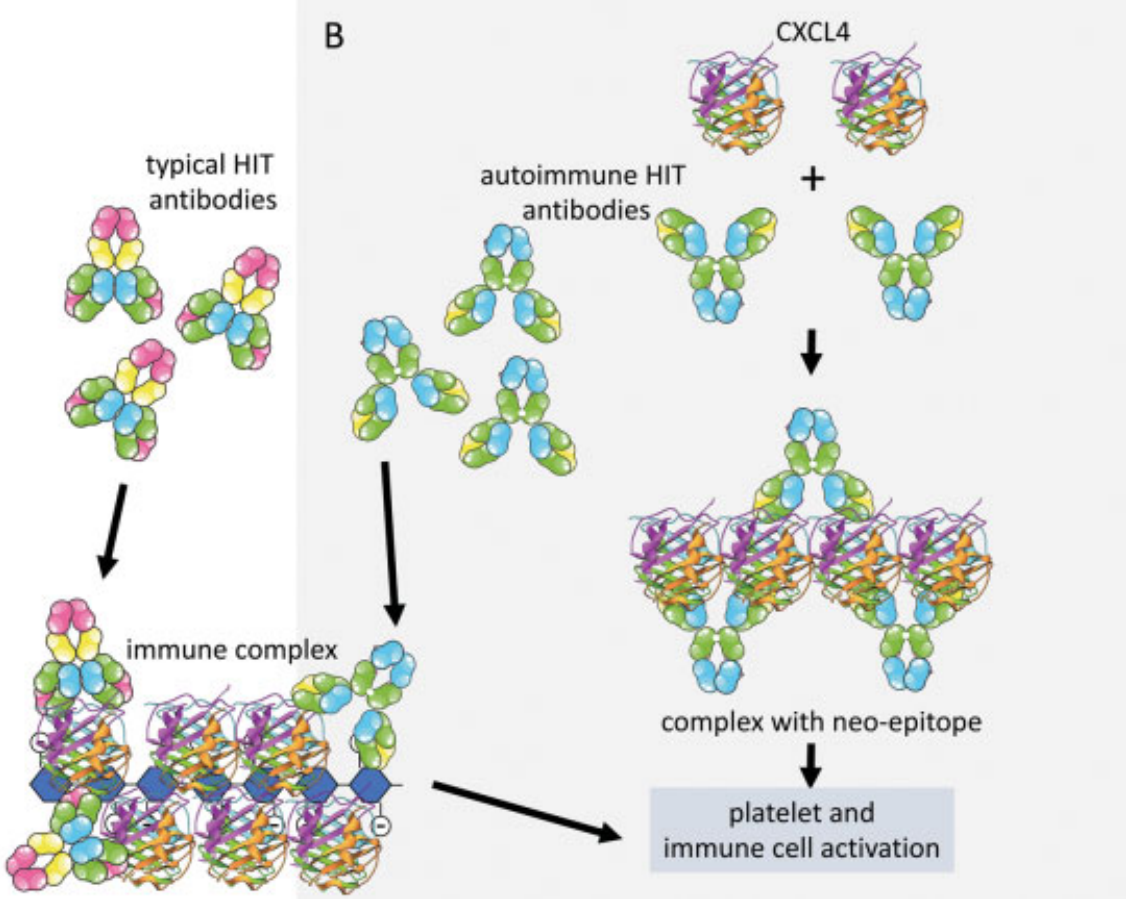

Fig. 1 Schematic representation of immune complex formation relevant for heparin-induced thrombocytopenia (HIT) pathophysiology. (A) Complexes of platelet factor 4 (CXCL4) and polyanions, e.g. heparin from large complexes with induction of conformational changes in CXCL4, thereby exposing neoepitopes. These complexes can directly activate B cells, which in turn produce antibodies that bind to CXCL4-heparin complexes. (B) Autoimmune HIT antibodies can bind CXCL4-heparin complexes but can additionally induce conformational changes in CXCL4 independently of polyanions. The resulting large complexes can directly activate platelets and might also activate immune cells, e.g. monocytes and $B$ cells.

patient autoimmune HIT antibodies. Solid phase binding assays further showed that the novel humAbs bound both to CXCL4 and CXCL4/heparin complexes. Interestingly, one of the humAbs showed dual reactivity toward CXCL4 and to heparin, which the authors explained by its positive zetapotential. By computational epitope mapping, the authors could predict that the binding epitope of the humAbs was spread over multiple monomer subunits within the CXCL4 tetramer and was distinct from the epitope of the monoclonal KKO. The observations above led the authors to conclude that the new humAbs, unlike the polyclonal ones from patients, can serve as a specific monoclonal source of model antibodies for the investigation of HIT and particularly autoimmune HIT pathophysiology.

The findings in this study are a further methodological step toward our understanding of the still incompletely characterized pathophysiology of typical HIT and autoimmune HIT. Given the potentially life-threatening consequences of HIT, there is a need for specific therapeutics, for example, that can disrupt HIT antibody-CXCL4 interactions. This is particularly relevant for autoimmune HIT, where treatment options are still limited. A thorough structural characterization of the (neo)epitopes of CXCL4 for HIT antibodies is an important first step for the development of specific drugs. Still, some issues remain unaddressed in this study. Most importantly, although interactions of CXCL4 with endogenous or bacterial polyanions (ribonucleic acid, polyphosphate, lipid A) are implicated in the pathophysiology of autoimmune HIT, ${ }^{13}$ the binding characteristics of the novel humAbs with these polyanions have not been assessed. Consequently, whether different polyanions induce similar neoepitopes in CXCL4 remains unanswered.

Taken together, the crooked conformation of CXCL4, induced by polyanions or directly by autoimmune HIT antibodies ultimately leading to symptoms ( also hold the key for developing specific therapeutics to prevent HIT in patients with heparin-based thromboprophylaxis risk.

\section{Funding}

The author received funding from the Landsteiner Foundation for Blood Transfusion Research (LSBR Nr. 1638) and the European Union's Horizon 2020 research and innovation program under the Marie Skłodowska-Curie grant agreement No. 722609 (INTRICARE) and TICARDIO (No. 813409). Single elements of the digital artwork were composed using elements of the Servier Medical Art collection, distributed under Creative Commons Attribution 3.0 Unported License.

Conflict of Interest

None declared.

\section{References}

1 Arepally GM, Ortel TL. Heparin-induced thrombocytopenia. Annu Rev Med 2010;61:77-90

2 Greinacher A. Heparin-induced thrombocytopenia. N Engl J Med 2015;373(19):1883-1884 
3 Vayne C, May MA, Bourguignon T, et al. Frequency and clinical impact of platelet factor 4-specific antibodies in patients undergoing extracorporeal membrane oxygenation. Thromb Haemost 2019;119(07):1138-1146

4 Martel N, Lee J, Wells PS. Risk for heparin-induced thrombocytopenia with unfractionated and low-molecular-weight heparin thromboprophylaxis: a meta-analysis. Blood 2005;106(08): 2710-2715

5 Gruel Y, Vayne C, Rollin J, et al. Comparative analysis of a French Prospective Series of 144 Patients with Heparin-Induced Thrombocytopenia (FRIGTIH) and the literature. Thromb Haemost 2020; 120(07):1096-1107

6 Carlson DS, Bartholomew JR, Gomes MP, McCrae KR, Chaturvedi S Outcomes of cardiovascular surgery utilizing heparin versus direct thrombin inhibitors in cardiopulmonary bypass in patients with previously diagnosed HIT. Thromb Haemost 2020;120(02): 300-305

7 Blanchet X, Cesarek K, Brandt J, et al. Inflammatory role and prognostic value of platelet chemokines in acute coronary syndrome. Thromb Haemost 2014;112(06):1277-1287
8 Kreimann M, Brandt S, Krauel K, et al. Binding of anti-platelet factor 4/heparin antibodies depends on the thermodynamics of conformational changes in platelet factor 4. Blood 2014;124(15): 2442-2449

9 Cai Z, Yarovoi SV, Zhu Z, et al. Atomic description of the immune complex involved in heparin-induced thrombocytopenia. Nat Commun 2015;6:8277

10 Zheng Y, Yu M, Podd A, et al. Critical role for mouse marginal zone B cells in PF4/heparin antibody production. Blood 2013;121(17): 3484-3492

11 Hui M, Sheppard JI, Li N, Warkentin TE. Neutrophil and monocyte counts in heparin-induced thrombocytopenia. Thromb Haemost 2019;119(06):941-951

12 Vayne C, Nguyen TH, Rollin J, et al. Characterization of new monoclonal PF4-specific antibodies as useful tools for studies on typical and autoimmune heparin-induced thrombocytopenia. Thromb Haemost 2020. Doi: 10.1055/s-0040-1717078

13 Greinacher A, Selleng K, Warkentin TE. Autoimmune heparininduced thrombocytopenia. J Thromb Haemost 2017;15(11): 2099-2114 